\title{
Identification of the metastasis potential and its associated genes in melanoma multinucleated giant cells using the PHA-ECM830 fusion method
}

\author{
RUIFANG MI ${ }^{1,2}$, CHUNXIAO PAN ${ }^{1,2}$, YIQIANG ZHOU ${ }^{1}$, YUANBO LIU ${ }^{1}$, GUISHAN JIN ${ }^{1}$ and FUSHENG LIU ${ }^{1}$ \\ ${ }^{1}$ Brain Tumor Research Center, Beijing Laboratory of Biomedical Materials, Beijing Neurosurgical Institute, \\ Beijing Tiantan Hospital Affiliated to Capital Medical University; ${ }^{2}$ Department of Pathology, \\ Institute of Basic Medical Sciences and School of Basic Medicine, Chinese Academy of \\ Medical Sciences and Peking Union Medical College, Beijing 100005, P.R. China
}

Received July 21, 2015; Accepted September 4, 2015

DOI: $10.3892 / o r .2015 .4376$

\begin{abstract}
Malignant melanoma causes skin cancer with high rates of mortality. Multinucleated giant cells (MGCs) are frequently observed in tumor pathological analysis, especially in metastasized sites, and are related to poor prognosis. However, the role of MGCs in melanoma development and metastasis is currently unknown. In the present study, we obtained melanoma MGCs (M-MGCs) in vitro using the modified phytohaemagglutinin (PHA)-ECM830 electronic fusion method (fusion efficiency was significantly enhanced by adding PHA to agglutinate cells before electronic fusion). We found that M-MGCs showed decreased proliferation potential but increased pulmonary metastasis ability relative to the parental B16-F10 cells. Microarray and bioinformatics analysis showed that $\beta$-tubulin gene group was significantly upregulated in M-MGCs. A member of this gene group, TUBB2B, exhibited significantly enhanced expression, indicating that it may play an important role in melanoma metastasis. Our results provide novel insights into the properties of melanoma and they could contribute towards the design of new strategies for rapid diagnosis and treatment of this cancer.
\end{abstract}

\section{Introduction}

Melanoma is a malignant tumor that forms in the skin and accounts for $75 \%$ of skin cancer mortality (1). High mortality

Correspondence to: Professor Fusheng Liu or Guishan Jin, Brain Tumor Research Center, Beijing Laboratory of Biomedical Materials, Beijing Neurosurgical Institute, Beijing Tiantan Hospital Affiliated to Capital Medical University, 6 Tiantan Xili Road, Dongcheng, Beijing 100050, P.R. China

E-mail: liufushengs@hotmail.com

E-mail: guishanjin7@163.com

Key words: melanoma multinucleated giant cells, metastasis, fusion method, $T U B B 2 B$ gene in melanoma cases is caused by the rapid proliferation rate of cells and their potential to metastasize at an early stage. For example, many melanomas have already metastasized to the lymph nodes and other organs by the time of diagnosis. Therefore, new strategies for rapid diagnosis and treatment of melanoma are urgently required because the effectiveness of treatment is highly dependent on the removal of the tumor at an early stage (2).

Multinucleated giant cells (MGCs) and polyploidy are commonly observed in cancer research and are related to poor clinical prognosis in, for example, breast cancer, prostate cancer and melanoma $(3,4)$. In pathologic analysis of MGCs, the cell and nuclear volumes appear enlarged and the chromosome number is heteroploid and polyploid (5). MGCs are often formed by cell fusion and fused cells are commonly found in metastatic tumor tissue (6).

Melanoma metastasis occurs mainly through hematogenous spread and is organ-specific, especially to the lungs, brain, skin and liver (7). Cancer metastasis requires a series of related processes to proceed. Cancer cells can spread via the blood or the lymphatic system to distant organs where tumor cells either adapt to the existing microenvironment or establish a new one by secreting growth factors or cytokines that facilitate their proliferation $(8,9)$. Specific genes play important roles in every step of metastasis. For example, genes that are specific to the circumstances, such as PTHRP, IL11, CSF $2 R B$ $(G M-C S F)$ and $T N F \alpha$, may primarily cause organ-specific metastasis $(10,11)$. However, the 'seed and soil' hypothesis also proposes that a tumor (seed) must adapt to the organ environment (soil) in order to survive and proliferate (12).

The cell fusion method is used in many fields (for example, in genome research and the generation of antibodies in vitro), where the polyethylene glycol (PEG) fusion has been the traditional method (13). Electrofusion method is used for high fusion efficiency. In order to achieve this high fusion efficiency in the BTX ECM2001 electronic fusion device, cells are arranged in a bead chain due to a high frequency alternating current wave (AC) that facilitates the cell membrane contact that is required for cell fusion and then fuse together using a direct current (DC). However, 
ECM2001 electronic fusion device is not so commonly used and are much more expensive (14). In this study, we obtained M-MGCs using a modified phytohaemagglutinin (PHA)-ECM830 electronic fusion method [with an ECM830 instrument for cell fusion and PHA for agglutination of cells, which facilitates cell membrane contact (15)].

Microarray analysis is a useful method for cancer study. Specific oligonucleotides or cDNA fragments are fixed to the gene chip, and the basic principle of which is hybridization (16). Tens of thousands of fragments can be detected simultaneously on gene chips such as the Affymetrix chip, which contains 22-base pair (bp) length oligonucleotides synthesized at high density using in situ lithography technology (17), making this process continuous, integrated and automatic (16).

In summary, we investigated the metastatic properties and gene expression of melanoma MGCs (M-MGCs). We found that they exhibited decreased proliferation potential but increased metastatic properties. Microarray analysis showed that the $\beta$-tubulin gene group (especially the $T U B B 2 B$ gene), which is related to cell invasion and metastasis, was significantly upregulated in M-MGC cells. Our results suggest the positive role of M-MGC cells in melanoma metastasis and the related genes in this process.

\section{Materials and methods}

Ethics statement. Animal research was carried out in strict accordance with the recommendations in the Guide for the Care and Use of Laboratory Animals of the Beijing Tiantan Hospital, Capital Medical University (no. 201201001). The Committee on the Ethics of Animal Experiments of the Beijing Tiantan Hospital, Capital Medical University, approved the experimental protocol. All efforts were made to minimize suffering of the animals.

Cell culture. Mouse melanoma cancer B16-F10 cells, M-MGCs, and 293T lentiviral packaging cell lines were all cultured in Dulbecco's modified Eagle's medium (DMEM) containing 5\% heat-inactivated fetal bovine serum (FBS) (both from HyClone, Carlsbad, CA, USA), $100 \mathrm{U} / \mathrm{ml}$ penicillin, and $0.1 \mathrm{mg} / \mathrm{ml}$ streptomycin, and in a humidified $5 \% \mathrm{CO}_{2}$ incubator at $37^{\circ} \mathrm{C}$.

Cell labeling. B16-F10 cells were labeled with pLL3.7 and pLL3.7-dsRED lentivirus. We purchased pLL3.7, which expresses EGFP alone, and the packaging plasmids (pMDL, pRev and pVSVG) from Addgene (Cambridge, MA, USA). The pLL3.7-dsRED plasmid was constructed by replacing an EGFP fragment of pLL3.7 with a polymerase chain reaction product of dsRED. Lentiviruses were generated from the 293T packaging cell line, and the B16-F10 cells were infected according to the natural protocol of lentivirus production and purification (18).

ECM830 cell fusion method. B16-F10-GFP $\left(1 \times 10^{7}\right)$ and B16-F10-RFP $\left(1 \times 10^{7}\right)$ cells were mixed and centrifuged at $900 \mathrm{rpm}$ for $5 \mathrm{~min}$. The cells were resuspended in $200 \mu \mathrm{l}$ of electronic liquid after the supernatant was completely removed. The cells were then shocked 3 times with $250 \mathrm{~V}$ for $30 \mu \mathrm{sec}$, rested for $5 \mathrm{~min}$, and finally placed in media for culture.
PHA-ECM830 cell fusion method. B16-F10-GFP $\left(1 \times 10^{7}\right)$ and B16-F10-RFP $\left(1 \times 10^{7}\right)$ cells were mixed, washed twice with DMEM, and incubated in $500 \mu 1$ of PHA in DMEM (50 $\mu \mathrm{g} / \mathrm{ml}$; Sigma-Aldrich, Carlsbad, CA, USA) at $37^{\circ} \mathrm{C}$ for $30 \mathrm{~min}$. After centrifugation (900 rpm, $5 \mathrm{~min}$ ) and complete removal of the supernatant, $200 \mu \mathrm{l}$ of electronic liquid was added. Cells were gently resuspended in DMEM and transferred to an electric shock cup where they were shocked 3 times with $250 \mathrm{~V}$ for $30 \mu \mathrm{sec}$. After resting for 5 min, cells were transferred to culture media.

Fluorescence-activated cell sorting (FACS). The cultured cells were washed twice and subjected to FACS using a BD FACSAria sorter with DMEM in order to purify GFP/RFP double-positive cells. The cells were collected in a medium containing $15 \%$ FBS and double antibiotics (100 U/ml penicillin, $0.1 \mathrm{mg} / \mathrm{ml}$ streptomycin (Gibco Life Technologies, Carlsbad, CA, USA), and then immediately cultured in $10 \%$ FBS DMEM.

Cell and nuclear volume measurements. Cells were suspended in DMEM at $1 \times 10^{5} / \mathrm{ml}$, and cell and nuclear volumes were detected and assessed using a Beckman Coulter counter multisizer 3 .

DNA content and karyotype analysis. Cells $\left(2 \times 10^{6}\right)$ were collected, washed twice with phosphate-buffered saline (PBS; HyClone), fixed with $75 \%$ ethanol, treated with $1 \mathrm{mg} / \mathrm{ml}$ RNase for $45 \mathrm{~min}$ at $37^{\circ} \mathrm{C}$, stained with $0.5 \mathrm{mg} / \mathrm{ml}$ propidium iodide (both from Sigma-Aldrich) for $45 \mathrm{~min}$ in the dark, and then analyzed for DNA content using flow cytometry. The DNA content of the subcutaneous and metastasis samples was analyzed after ex vivo culture. For karyotype analysis, cells were sent to the Department of Medical Genetics of the Institute of Basic Medical Sciences (IBMS), treated with colcemid and stained with Giemsa solution (both from Sigma-Aldrich).

In vitro proliferation rate curve. Cells $\left(2 \times 10^{5}\right)$ were plated on $60 \mathrm{~mm}$ plates for proliferation rate analysis. The medium was changed every 2 days and the number of cells was counted daily.

Tumor xenografts and analysis. We used C57BL/6 mice (female, 20-25 g, 8-week old; Cancer Institute, Chinese Academy of Medical Sciences, Beijing, China) in all the experiments. In the subcutaneous studies, cells $\left(5 \times 10^{4}\right)$ in PBS were injected subcutaneously into the rear flank of the mice in order to determine tumor growth rate in vivo. The animals were observed daily and tumor volumes were measured every 5 day using a vernier caliper. The mice were euthanized when tumor volumes exceeded $2,000 \mathrm{~mm}^{3}$ or when $>20 \%$ of their body mass was lost (measured by using an electronic balance).

In the metastasis studies, cells $\left(1 \times 10^{4}\right)$ in PBS were injected intravenously into the mice, which were then observed daily. They were euthanized 25 days after this injection or when they showed signs of morbidity (anorexia, difficulty moving, and/or weight loss $>20 \%$ ). Pulmonary metastatic samples were collected and then measured using an electronic balance (Mettler Toledo AL204). 

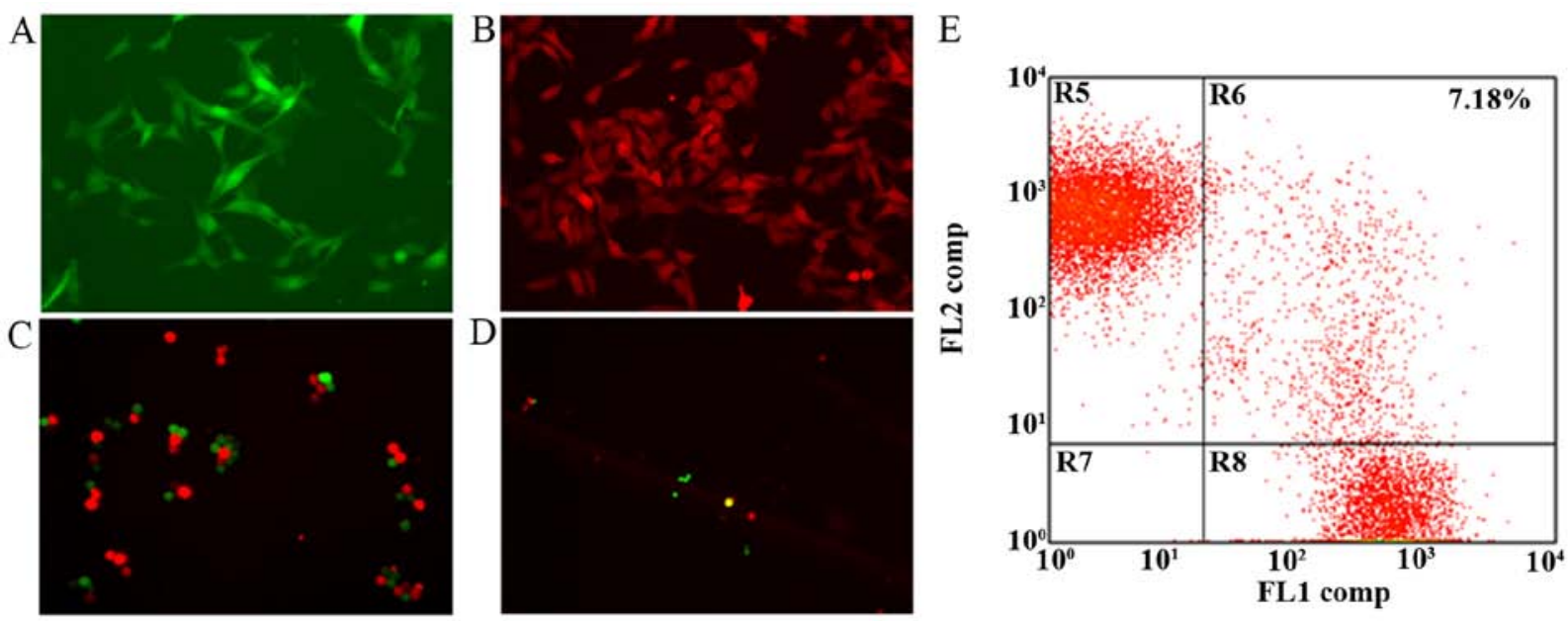

Figure 1. A modified PHA-ECM830 electronic fusion method was used to obtain melanoma multinucleated giant cells. (A and B) Melanoma cells were labeled with (A) GFP and (B) RFP fluorescence using lentivirus (x400). (C) The B16-F10 cells agglutinated after the addition of PHA (x200). (D) Fusion cells exhibited GFP and RFP simultaneously (observed as yellow when overlaid) after cell fusion. (E) Melanoma fusion cells were analyzed and selected by using FACS.

Histological analysis. Tumor samples and lung tissues were excised, fixed in $10 \%$ neutral-buffered formalin and embedded in paraffin for hematoxylin and eosin (HE) staining at the Pathology Centre of the IBMS.

RNA extraction and gene chip analysis. RNA was extracted using TRIzol reagent (Invitrogen Life Technologies, Carlsbad, CA, USA) according to the manufacturer's suggested protocol. RNA samples were then sent to the central laboratory of the IBMS for subsequent Affymetrix gene chip analysis. A gene expression ratio of $\mathrm{M}-\mathrm{MGCs} / \mathrm{B} 10-\mathrm{F} 10 \geq 2$ was considered significant. Gene functions were analyzed using Ingenuity pathway analysis (IPA) database software.

Western blotting analysis. Cells were harvested in lysis buffer (2\% SDS, $0.1 \mathrm{~mol} / 1 \mathrm{DTT}, 10 \%$ glycerol and $60 \mathrm{mmol} / \mathrm{l}$ Tris, $\mathrm{pH}$ 6.8) and boiled at $98^{\circ} \mathrm{C}$ for $10 \mathrm{~min}$. The protein lysates were separated by using 4-12\% Bis-Tris SDS-PAGE gel (Invitrogen Life Technologies) and transferred onto polyvinyl difluoride (PVDF) membranes (Pierce Chemical Co., Rockford, IL, USA). Non-specific reactivity was blocked by $10 \%$ non-fat dry milk in TBST buffer $(20 \mathrm{mmol} / \mathrm{l}$ Tris- $\mathrm{HCl}, 150 \mathrm{mmol} / \mathrm{l} \mathrm{NaCl}$, $0.05 \%$ Tween-20, $\mathrm{pH} 7.5$ ) at room temperature for $2 \mathrm{~h}$. The membranes were then incubated with the primary antibodies for TUBB2B (1:500) and phospho-S6 (1:4,000) (both from Abcam, Cambridge, UK), and $\beta$-actin $(1: 8,000)$ at $4^{\circ} \mathrm{C}$ for $10 \mathrm{~h}$, followed by another incubation with a horseradish peroxidase (HRP)-conjugated secondary antibody (both from Santa Cruz Biotechnology, Santa Cruz, CA, USA) at 1:5,000 dilution and at room temperature for $4 \mathrm{~h}$.

Statistical analysis. All data are presented as the mean \pm SD. Data were analyzed, and P-values produced, using SPSS 17.0 statistical software. $\mathrm{P}<0.05$ was considered to indicate a statistically significant difference.

\section{Results}

M-MGCs obtained by a modified PHA-ECM830 electronic fusion method. B16-F10 is a commonly used melanoma cell line with a high proliferation rate and metastatic potential to the lungs when injected intravenously (19). We used these cells to obtain M-MGCs by using cell fusion in vitro. First, B16-F10 cells were labeled with GFP and RFP fluorescence (Fig. 1A and B) for easy screening of the after fusion. Initially, we attempted to fuse the labeled B16-F10 cells using the ECM830 electronic fusion method; however, the cell fusion efficiency was so limited that positive fusion cells (those that showed both GFP and RFP and appeared yellow after overlay) were only partially observed in one microscopic field. Therefore, we added the cell-agglutinating agent PHA prior to cell fusion in order to enhance the level of cell membrane contact, which is an essential part of the process (Fig. 1C). The addition of PHA significantly increased fusion efficiency, and fusion cells were clearly observable in one field (Fig. 1D). FACS analysis showed that the fusion efficiency was $\leq 7.18 \%$ (Fig. 1E). The presence of live fusion cells after selection indicated successful cell fusion.

The cell and nuclear sizes of M-MGCs were both twice as large as those of the parent B16-F10 cells (Figs. 1D and 2B). DNA content and karyotype analysis showed that the obtained M-MGCs had converted from being hyperdiploid to hypertetraploid (Fig. 2A and $\mathrm{C}$ ).

M-MGCs exhibit increasedmetastatic potential. The M-MGCs showed a decreased rate of proliferation in the cell culture. Both their proliferation rate in vitro (Fig. 3A) and the growth rate in vivo (Fig. 3B) decreased. However, the downregulation of the M-MGCs growth rate in vivo was not that obvious compared to the change of the proliferation rate in vitro. This discrepancy may have arisen because of the enlarged tumor size of the M-MGCs. After the tumor cells were injected intravenously, the potential for M-MGCs to metastasize to the lung was enhanced (Fig. 4A). The weight of lung containing metastatic tumors and the metastatic nodules on the lung surface were counted, and the results also showed the enhanced metastatic potential of M-MGCs to the lungs (Fig. 4B and C). Hematoxylin-eosin (HE) analysis showed the metastatic M-MGCs to the lungs (Fig. 4D). However, HE analysis also showed the M-MGCs did not metastasize to other organs, such as the liver, kidneys, spleen and brain. 

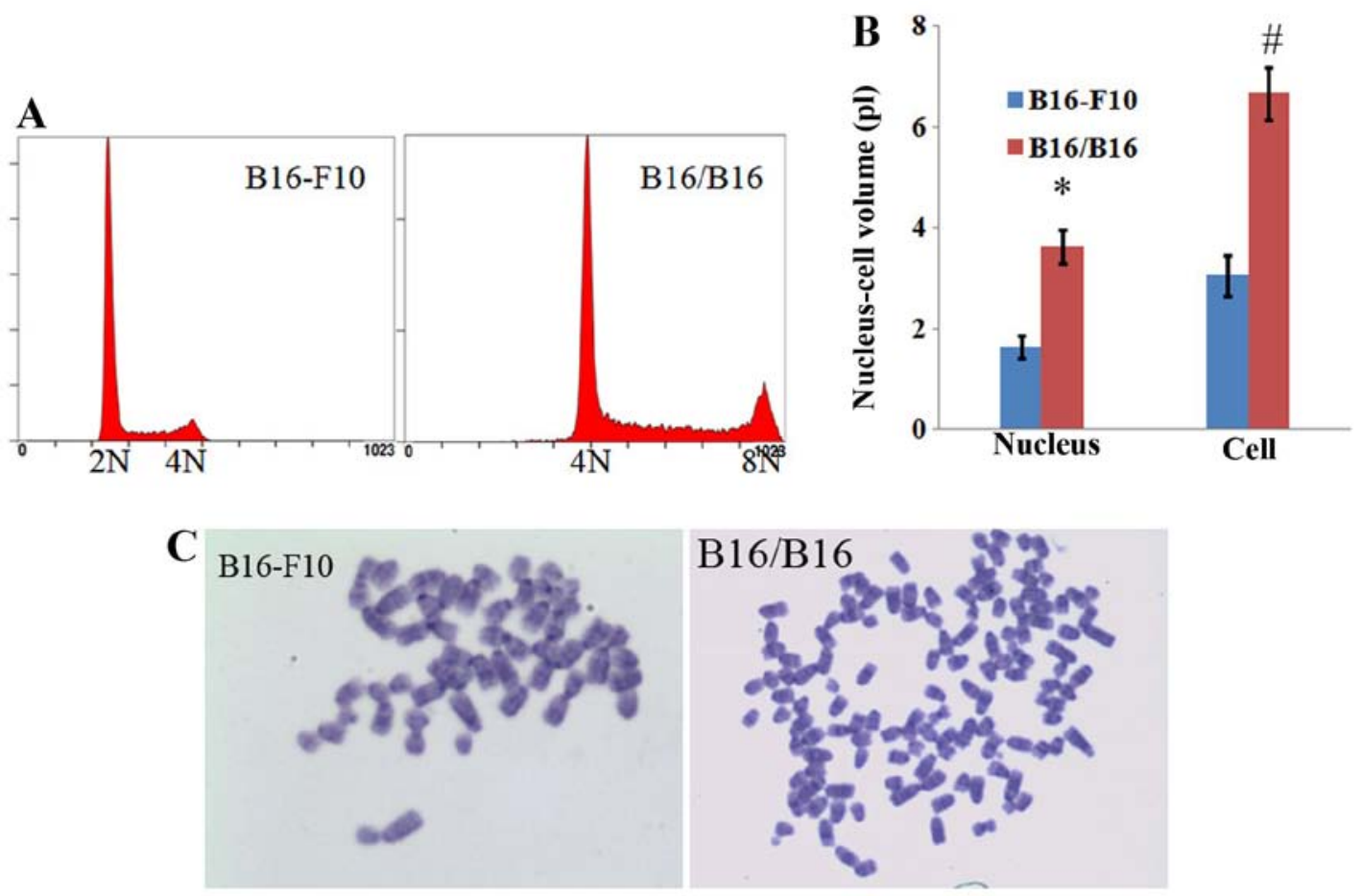

Figure 2. Melanoma multinucleated giant cells (M-MGCs) show double the DNA content and enlarged volumes compared to parental B16-F10 cells. (A) The DNA content of the M-MGCs was almost twice that of the parental cells. (B) The M-MGCs acquired enlarged nuclei and cell volumes. (C) Karyotyping analysis of the M-MGCs and the parental B16-F10 cells $\left({ }^{*}, * \mathrm{P}<0.05\right.$, compared to the B16-F10 samples). Data are representative of three experiments, and each assay was performed in triplicate.


Figure 3. Melanoma multinucleated giant cells (M-MGCs) exhibited reduced proliferation rate. (A and B) The proliferation rate of M-MGCs reduced in vitro (A) and the growth rate reduced in vivo (B). ("P<0.05, compared to the B16-F10 samples). The data are representative of three experiments, and each assay was performed in quintuplicate.

Microarray analysis show changes to metastasis in M-MGCs associated with $\beta$-tubulin gene group. The A260/280 ratio of extracted RNA was $\sim 1.8$. There were clear $28 \mathrm{~S}, 18 \mathrm{~S}$ and $5 \mathrm{~S}$ RNA bands in the $1.2 \%$ formaldehyde agarose gel, and the $28 \mathrm{~S} / 18 \mathrm{~S}$ ratio was $\sim 2$, which indicated the integrity of the RNA fragments. Microarray analysis showed that changes in the AKT network were associated with proliferation and metastasis in the M-MGCs (Fig. 5). At the centre of this network, the AKT gene was significantly downregulated. Other genes in the PI3K-AKT pathway that play an important role in cell proliferation, such as PI3K and pS6, were also downregulated (Table I). Western blot analysis confirmed the downregulation of pS6, downstream of the PI3K-AKT pathway in M-MGCs (Fig. 6A and C). The $\beta$-tubulin gene group, which is related to cell migration and invasion, was upregulated in M-MGCs (Table II). The TUBB2B gene, which is also part of the AKT network (Fig. 5), was significantly upregulated in M-MGCs, and this was also confirmed by western blot analysis (Fig. 6A and B).

M-MGCs show DNA stability following culture in vitro and ex vivo. M-MGCs were cultured for 6 months ex vivo after 

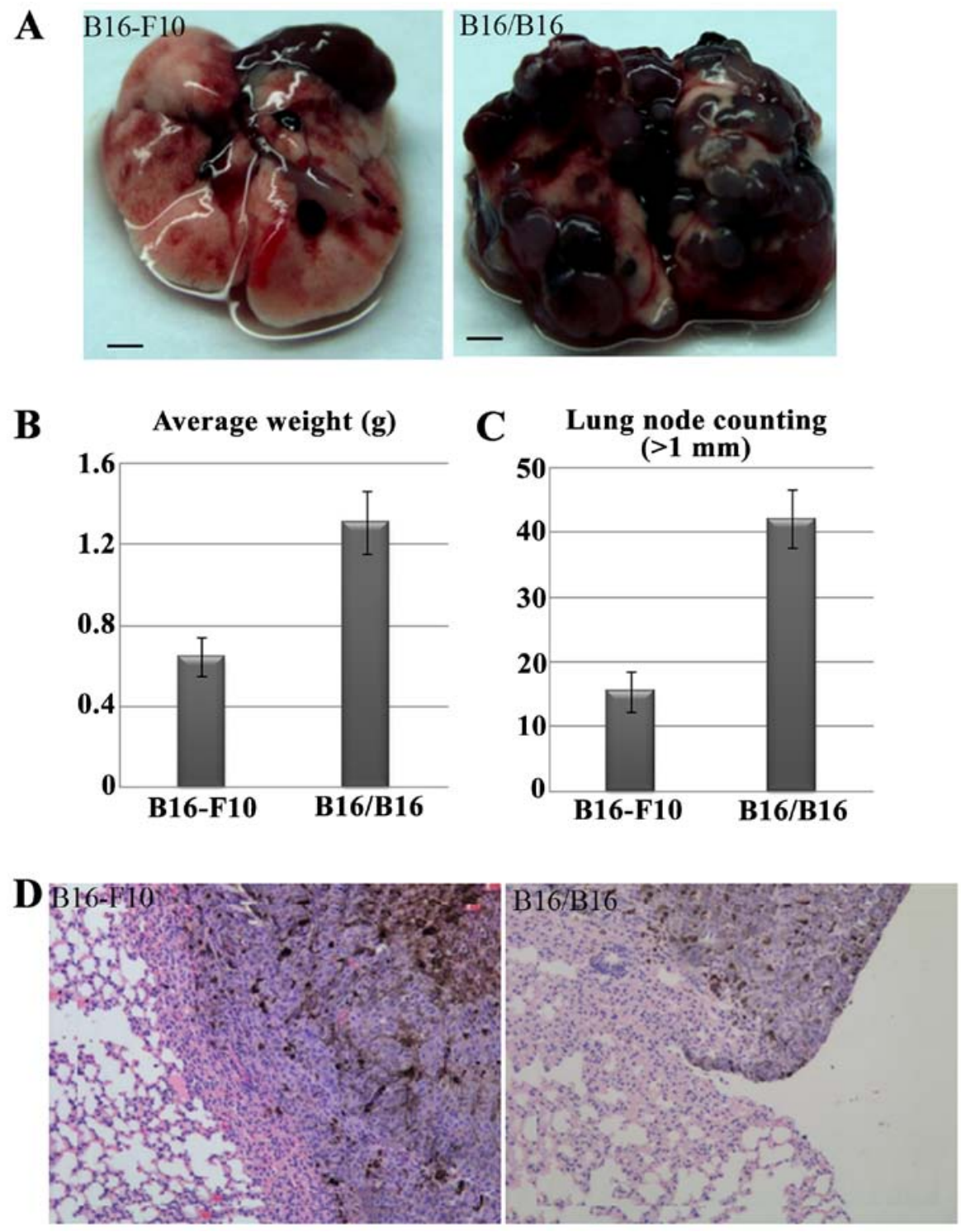

Figure 4. Melanoma multinucleated giant cells (M-MGCs) exhibit enhanced metastatic ability. (A) Pathological anatomy analysis of lung tissue with metastatic M-MGCs and B16-F10 cells (bars represent $1.5 \mathrm{~mm}$ ). (B and C) Metastasized lung weight (B) and the number of lung nodules (C) produced with M-MGCs was considerably higher than those produced with B16-F10 cells. (D) HE staining of lung tissue with metastatic M-MGCs and B16-F10 cells (x100). ( ${ }^{* * \# P} \mathrm{P}<0.05$, compared to the B16-F10 samples). The data are representative of three experiments, and each assay was performed in quintuplicate.

Table I. The PI3K-AKT pathway is downregulated in melanoma multinucleated giant cells.

\begin{tabular}{llr}
\hline Gene symbol & \multicolumn{1}{c}{ Gene title } & Fold-change \\
\hline Pik3r1 & $\begin{array}{l}\text { Phosphatidylinositol 3-kinase, } \\
\text { regulatory subunit, polypeptide 1 (p85 } \alpha)\end{array}$ & $-2.05297^{\mathrm{a}}$ \\
Akt3 & $\begin{array}{l}\text { Thymoma viral proto-oncogene 3 } \\
\text { Rps6ka1 }\end{array}$ & $-2.65325^{\mathrm{a}}$ \\
\hline
\end{tabular}

${ }^{\mathrm{a}} \mathrm{P}<0.01$, compared with the B16-F10 parental cell group; $\mathrm{n}=2$.

being derived from metastatic tumor sites. DNA content analysis showed that the genotype of M-MGCs was consistent after ex vivo and in vitro passage, indicating the genomic stability of M-MGCs (Fig. 7).
Table II. The $\beta$-tubulin gene group was upregulated in melanoma multinucleated giant cells

\begin{tabular}{llc}
\hline Gene symbol & \multicolumn{1}{c}{ Gene title } & Fold-change \\
\hline Tubb2b & Tubulin, $\beta 2 \mathrm{~b}$ & $10.8058^{\mathrm{a}}$ \\
Tubb6 & Tubulin, $\beta 6$ & $3.39871^{\mathrm{a}}$ \\
Tubb2a-ps2 & Tubulin, $\beta 2 \mathrm{a}$, pseudogene 2 & $2.82951^{\mathrm{a}}$ \\
\hline
\end{tabular}

${ }^{\mathrm{a}} \mathrm{P}<0.01$, compared with the $\mathrm{B} 16-\mathrm{F} 10$ parental cell group; $\mathrm{n}=2$.

\section{Discussion}

MGCs are commonly observed in melanoma pathological analysis, especially at metastatic tumor sites, and they are usually associated with poor prognosis $(3,4,20)$. Cell fusion, which is considered a cause of cancer metastasis, is one 


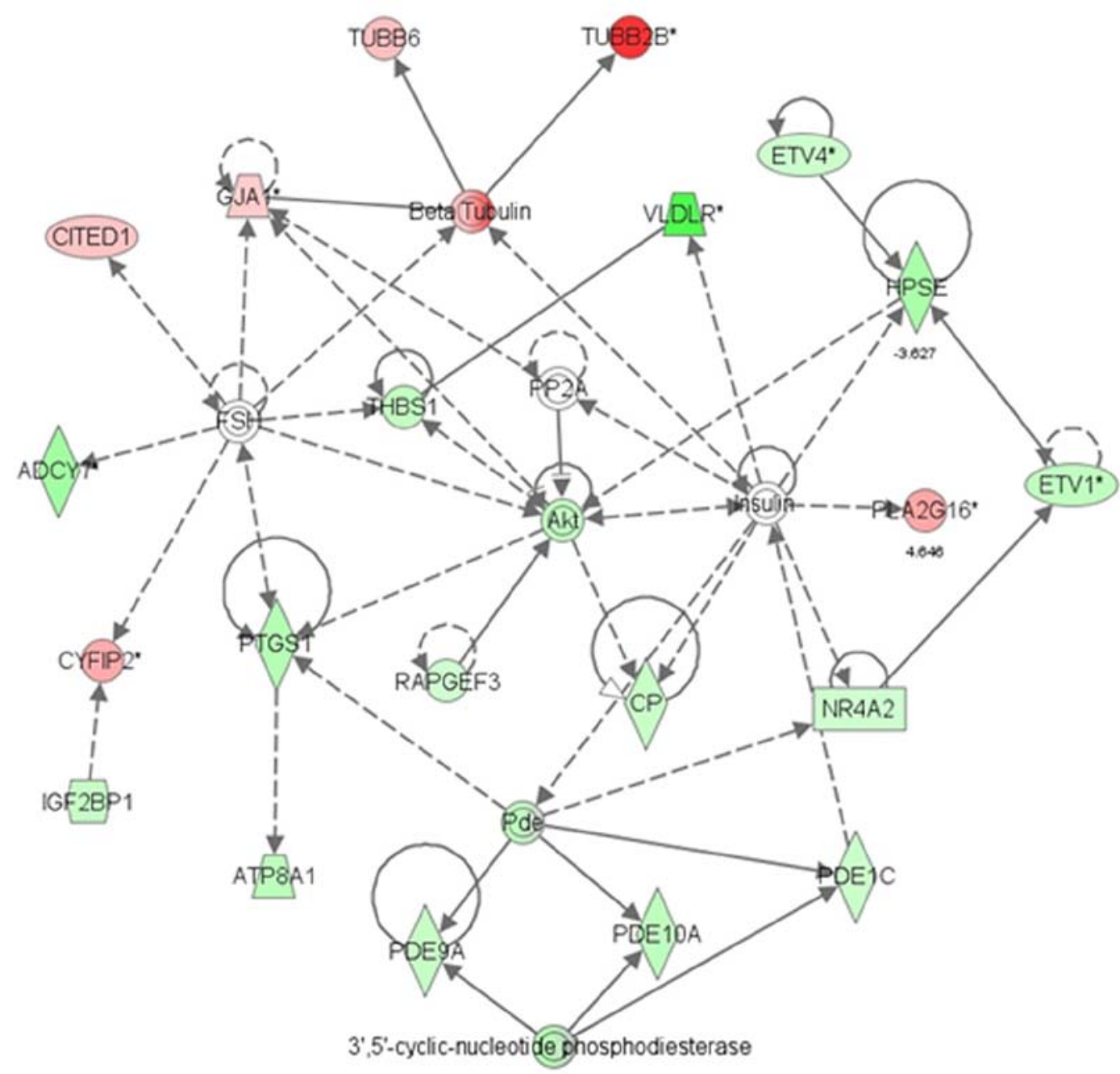

Figure 5. The AKT network in melanoma multinucleated giant cells (M-MGCs). Gene expression change of genes in the AKT network in M-MGCs.

A $\quad$ B16/F10 $\quad B 16 / B 16$

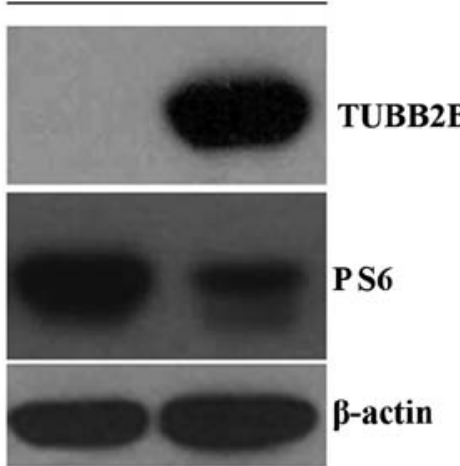

B

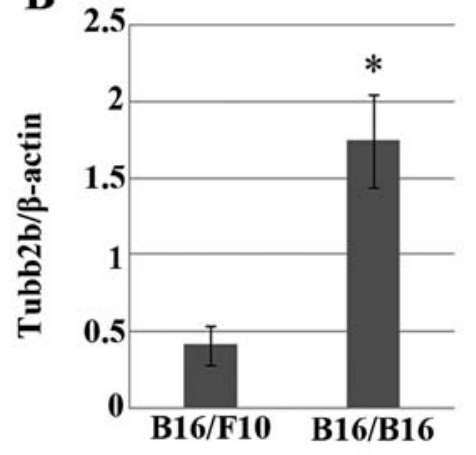

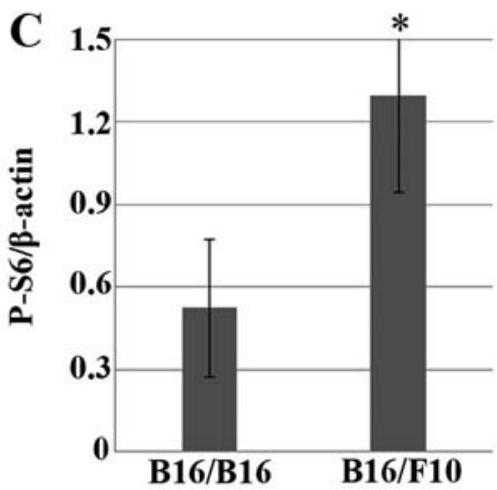

Figure 6. TUBB2B and pS6 gene expression in melanoma multinucleated giant cells. Whole cell extracts were used to detect the activity of $T U B B 2 B$ (A and B) and phosphor-S6 by western blotting (A and $\mathrm{C}$ ). ( $\mathrm{P}<0.05$, compared to the B16-F10 samples). Data are representative of three experiments, and each assay was performed in triplicate.

process by which MGCs are formed (20). For the formation of MGCs, cancer cells can fuse with many types of somatic cells, including stromal and epithelial cells, as well as other cancer cells. In the present study, we obtained the MGCs in vitro by fusing melanoma cells with each other (21-23).

In the present study, an ECM830 electronic fusion device was used to achieve melanoma cell fusion. However, the fusion efficiency was very limited, and so PHA was used to promote contact between the cells by agglutination, thereby promoting cell fusion (13). PHA significantly increased fusion efficiency ( $>10$ times); therefore, our study demonstrates a convenient and effective fusion method (PHA-ECM830 fusion) that could be used for future cancer research. The MGCs obtained by this method could be used, for example, 


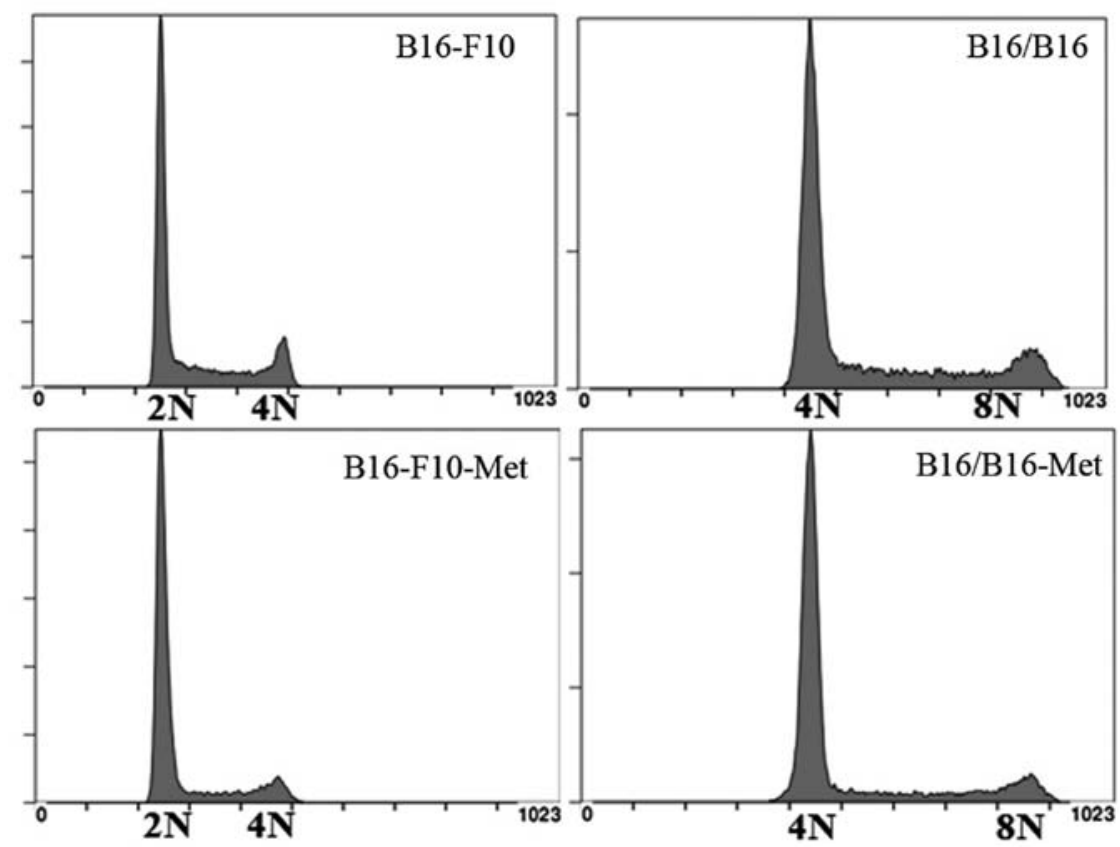

Figure 7. The genome stability of melanoma multinucleated giant cells (M-MGCs) after prolonged ex vivo passage. Pulmonary metastasis samples were analyzed after ex vivo culture for 6 months. The data are representative of three experiments, and each assay was performed in triplicate.

in studies of organ-specific cancer metastasis and genome stability research. We also found that the MGCs obtained in this study exhibited stable genome characteristics. Although the mechanism of this property was not revealed in our study and awaits further research, these fusion cells could potentially provide a useful model for genome stability research.

Cell proliferation and metastasis are two of the most important characteristics of cancer cells. However, previous studies have shown that proliferation rate is reduced when cancer cells are fused with fibroblast cells (24). In our study, the M-MGCs we obtained showed decreased proliferation and increased metastasis to the lungs. These effects could be due to tumor suppressor genes, such as p53 $(24,25)$. Another possible explanation is that a decreased rate of proliferation enables cancer cells to execute other functions, such as cancer metastasis. However, the function of MGCs in cancer malignancy is complicated, and further research will be necessary to validate these hypotheses.

Our microarray analysis indicated that the PI3K-AKT pathway includes the primary set of differentially expressed genes associated with cell proliferation in M-MGCs. We also found decreased protein expression in this pathway, indicating that it probably plays an important role in melanoma cell proliferation. In the PI3K-AKT pathway, the upstream molecules RTK-PI3K-AKT activate the downstream molecules mTOR-pS6 and further promote the eukaryotic initiation factor 4E (eIF4E) to regulate protein translation (26). AKT can also directly act on transcription factors, such as p27 and p21, to regulate the cell cycle and cell proliferation. There are many specific inhibitors in this pathway; for example, wortmannin and rapamycin specifically inhibit the activity of PI3K and mTOR, respectively (26-28). The PI3K-AKT pathway is highly active in melanoma cells, which proliferate rapidly (27), and AKT3 reportedly significantly inhibits the proliferation rate of these cells.
Cancer metastasis involves a series of processes, including the metastasis of the primary melanoma cells to a distant organ through blood or lymph vessels where they adhere to the capillary and invade the new environment. The cancer cells adapt to the new environment and promote cell proliferation by secreting cell growth factors or cytokines (9). In our study, metastasis of melanoma was specific to the lungs, and this may be because MGCs only express lung-specific metastasis genes and are, therefore, a good fit for the lung environment. In future studies, it will be necessary to investigate the mechanisms of cell adhesion, cell invasion, and cell growth in the new organ environment.

Microtubulin is a part of the globular protein family, of which $\alpha$-tubulin and $\beta$-tubulin are members. These two proteins have a similar dimensional structure and they form tightly integrated dimers to become the subunits of microtubules. $\beta$-tubulin can be constructed by several subunits including TUBB, TUBB1, TUBB2A, TUBB2B and TUBB2C, of which TUBB2B plays an important role in cell invasion and migration. In Drosophila, salivary gland development is inhibited when the $T U B B 2 B$ gene is knocked out (29). In rats, knockdown of $T U B B 2 B$ inhibits the migration of the cortical neurons (30). In our study, $T U B B 2 B$ was significantly upregulated in M-MGCs, which indicates that it may play an important role in melanoma metastasis.

Our study demonstrates the changes in cell metastatic ability in M-MGCs are associated with $\beta$-tubulin gene group. However, the specific genes for the metastasis of melanoma cells and M-MGCs are not explicit, further study can be done to verify the results. For the organ specific metastasis, cell fusion method is a special useful tool (for example, different organ specific metastatic cells can be fused together to see the genotype and the following metastatic phenotype changes of the fusion cells). Collectively, our results provide novel insights into the properties of melanoma and they could contribute 
towards the design of new strategies for rapid treatment of this cancer.

\section{Acknowledgements}

The present study was supported by a grant from the National Natural Science Foundation of China (nos. 81302186, 81372354 and 81271563), the Beijing Natural Science Foundation (nos. 7132034 and 7151002), the Beijing Health System High-level Personnel Building Foundation (no. 2013-3-018), the Beijing Laboratory of Biomedical Materials Foundation and the Natural Science Foundation of Beijing Neurosurgical Institute. We thank Professor Hongbing Zhang, Professor Yuqin Liu and Jianhui Ma for their helpful advice and support. We also would like to thank Editage (www.editage.com) for English language editing.

\section{References}

1. DeSantis CE, Lin CC, Mariotto AB, Siegel RL, Stein KD, Kramer JL, Alteri R, Robbins AS and Jemal A: Cancer treatment and survivorship statistics, 2014. CA Cancer J Clin 64: 252-271, 2014.

2. Damsky WE, Theodosakis $\mathrm{N}$ and Bosenberg M: Melanoma metastasis: New concepts and evolving paradigms. Oncogene 33: 2413-2422, 2014.

3. Abdalla F, Boder J, Markus R, Hashmi H, Buhmeida A and Collan Y: Correlation of nuclear morphometry of breast cancer in histological sections with clinicopathological features and prognosis. Anticancer Res 29: 1771-1776, 2009.

4. Rajagopalan $\mathrm{H}$ and Lengauer C: Aneuploidy and cancer. Nature 432: 338-341, 2004

5. Mossbacher U, Knollmayer S, Binder M, Steiner A, Wolff K and Pehamberger $\mathrm{H}$ : Increased nuclear volume in metastasizing 'thick' melanomas. J Invest Dermatol 106: 437-440, 1996.

6. Pawelek JM and Chakraborty AK: Fusion of tumour cells with bone marrow-derived cells: A unifying explanation for metastasis. Nat Rev Cancer 8: 377-386, 2008.

7. Fidler IJ: The role of the organ microenvironment in brain metastasis. Semin Cancer Biol 21: 107-112, 2011.

8. Langley RR and Fidler IJ: The seed and soil hypothesis revisited - the role of tumor-stroma interactions in metastasis to different organs. Int J Cancer 128: 2527-2535, 2011.

9. Nguyen DX, Bos PD and Massagué J: Metastasis: From dissemination to organ-specific colonization. Nat Rev Cancer 9: 274-284, 2009.

10. Nguyen DX and Massagué J: Genetic determinants of cancer metastasis. Nat Rev Genet 8: 341-352, 2007.

11. Gupta GP, Minn AJ, Kang Y, Siegel PM, Serganova I, Cordón-Cardo C, Olshen AB, Gerald WL and Massagué J: Identifying site-specific metastasis genes and functions. Cold Spring Harb Symp Quant Biol 70: 149-158, 2005.
12. Fidler IJ: The pathogenesis of cancer metastasis: The 'seed and soil' hypothesis revisited. Nat Rev Cancer 3: 453-458, 2003.

13. Lentz BR: PEG as a tool to gain insight into membrane fusion. Eur Biophys J 36: 315-326, 2007.

14. Kandušer M and Ušaj M: Cell electrofusion: Past and future perspectives for antibody production and cancer cell vaccines. Expert Opin Drug Deliv 11: 1885-1898, 2014.

15. Veselý P, Entlicher G and Kocourek J: Pea phytohemagglutinin selective agglutination of tumour cells. Experientia 28: 1085-1086, 1972.

16. Russo G, Zegar C and Giordano A: Advantages and limitations of microarray technology in human cancer. Oncogene 22: 6497-6507, 2003

17. Dalma-Weiszhausz DD, Warrington J, Tanimoto EY and Miyada CG: The affymetrix GeneChip platform: An overview. Methods Enzymol 410: 3-28, 2006.

18. Kutner RH, Zhang XY and Reiser J: Production, concentration and titration of pseudotyped HIV-1-based lentiviral vectors. Nat Protoc 4: 495-505, 2009.

19. Fidler IJ and Nicolson GL: Organ selectivity for implantation survival and growth of B16 melanoma variant tumor lines. J Natl Cancer Inst 57: 1199-1202, 1976.

20. Wang X, Willenbring H, Akkari Y, Torimaru Y, Foster M, Al-Dhalimy M, Lagasse E, Finegold M, Olson S and Grompe M: Cell fusion is the principal source of bone-marrow-derived hepatocytes. Nature 422: 897-901, 2003.

21. Chakraborty AK, Sodi S, Rachkovsky M, Kolesnikova N, Platt JT, Bolognia JL and Pawelek JM: A spontaneous murine melanoma lung metastasis comprised of host $\mathrm{x}$ tumor hybrids. Cancer Res 60: 2512-2519, 2000.

22. Duelli D and Lazebnik Y: Cell-to-cell fusion as a link between viruses and cancer. Nat Rev Cancer 7: 968-976, 2007.

23. Duelli D and Lazebnik Y: Cell fusion: A hidden enemy? Cancer Cell 3: 445-448, 2003.

24. Harris $\mathrm{H}$ : The analysis of malignancy by cell fusion: The position in 1988. Cancer Res 48: 3302-3306, 1988.

25. Green DR and Kroemer G: Cytoplasmic functions of the tumour suppressor p53. Nature 458: 1127-1130, 2009.

26. Babchia N, Calipel A, Mouriaux F, Faussat AM and Mascarelli F: The PI3K/Akt and mTOR/P70S6K signaling pathways in human uveal melanoma cells: Interaction with B-Raf/ERK. Invest Ophthalmol Vis Sci 51: 421-429, 2010.

27. Cantley LC: The phosphoinositide 3-kinase pathway. Science 296: 1655-1657, 2002.

28. Wong KK, Engelman JA and Cantley LC: Targeting the PI3K signaling pathway in cancer. Curr Opin Genet Dev 20: 87-90, 2010.

29. Jattani R, Patel U, Kerman B and Myat MM: Deficiency screen identifies a novel role for beta 2 tubulin in salivary gland and myoblast migration in the Drosophila embryo. Dev Dyn 238: 853-863, 2009

30. Jaglin XH, Poirier K, Saillour Y, Buhler E, Tian G, Bahi-Buisson N, Fallet-Bianco C, Phan-Dinh-Tuy F, Kong XP, Bomont $\mathrm{P}$, et al: Mutations in the beta-tubulin gene TUBB2B result in asymmetrical polymicrogyria. Nat Genet 41: 746-752, 2009. 